\title{
OPTIMAL BLIND CARRIER SYNCHRONIZATION FOR M-PSK BURST TRANSMISSIONS
}

\author{
Y. Wang ${ }^{1}$, E. Serpedin ${ }^{1}$ and P. Ciblat ${ }^{2}$ \\ ${ }^{1}$ Dept. of Electrical Engineering, Texas A\&M University, College Station, TX 77843, USA \\ ${ }^{2}$ Ecole Nationale Supérieure des Télécommunications, Paris, France
}

\begin{abstract}
This paper introduces a family of blind feedforward nonlinear estimators for joint estimation of carrier phase, frequency offset and Doppler rate for burst-mode phase shift keying (PSK) transmissions. An optimal or "matched" nonlinear estimator that exhibits the smallest asymptotic variance within the family of envisaged estimators is developed. The asymptotic performance of these estimators is established in closed-form expression and compared with the Cramèr-Rao lower bound for an unmodulated carrier. Finally, computer simulations are presented to corroborate the theoretical performance analysis.
\end{abstract}

\section{INTRODUCTION}

Non-data aided (NDA) or blind feedforward carrier synchronization of burst M-PSK transmissions has received considerable attention in the literature. A generalized form of the Maximum Likelihood Feedforward (ML FF) algorithm was originally proposed by A. J. Viterbi and A. M. Viterbi as a blind carrier phase estimator for fully modulated M-PSK transmissions, and it exhibits improved performance at low and intermediate Signal-to-Noise Ratios (SNRs) [9], [8]. This carrier phase estimator is referred to as the Viterbi and Viterbi (V\&V) algorithm [4], and has been partially extended in [2] to a family of blind frequency offset estimators for burst M-PSK modulations. Reference [4] introduces another non-data aided feedforward carrier frequency offset estimator that relies on a rather restrictive set of assumptions and whose asymptotic variance is evaluated based on several approximations. It is interesting to remark that [7] proposes a quite general nonlinear blind least-squares (NLS) estimator, but its performance was neither analyzed nor optimized.

In this paper, a family of blind feedforward joint carrier phase, frequency offset and Doppler rate estimators for carriers that are fully modulated by M-PSK modulations is proposed based on the V\&V algorithm. An optimal or "matched" nonlinear estimator that achieves the smallest asymptotic (large sample) variance within the family of blind NLS estimators is also proposed. Optimal monomial approximations of the matched estimator are also developed. The goal of the present paper is to provide a thorough and rigorous analysis of the statistical properties of the proposed family of nonlinear carrier synchronizers, and to propose estimators with improved performance.

As we shall see, the proposed family of estimators presents high convergence rates, provides high accurate estimates for phase, frequency offset and Doppler rate, and admits low complexity digital implementations, without being necessary to oversample (or fractionally-sample) the received signal faster than the Nyquist rate. The performance of these algorithms achieves closely the Cramèr-Rao bound (CRB) of an unmodulated carrier at medium and high SNRs.

\section{PROBLEM FORMULATION}

Consider the baseband representation of an M-PSK modulated signal transmitted through an AWGN channel. Assume that filtering is evenly split between transmitter and receiver so that the overall channel is Nyquist. Filtering the received waveform through a matched filter and sampling at the right time instants yields:

$$
\begin{aligned}
& x(n)=w(n) e^{j \phi(n)}+v(n), n=0, \ldots, N-1, \\
& \phi(n)=\theta+2 \pi F_{e} T n+\eta T^{2} n^{2},
\end{aligned}
$$

where $\{w(n)\}$ is the sequence of zero-mean unit variance $\left(\sigma_{w}^{2}:=\right.$ $\mathrm{E}\left\{|w(n)|^{2}\right\}=1$ ) independently and identically distributed (i.i.d.) M-PSK symbols, $\theta, F_{e}$ and $\eta$ stand for carrier phase, frequency offset and Doppler rate, respectively, $T$ denotes the symbol period, and $\{v(n)\}$ is a zero-mean white Gaussian noise process independent of $w(n)$ and with variance $\sigma_{v}^{2}:=\mathrm{E}\left\{|v(n)|^{2}\right\}$. The Signal-to-Noise Ratio is defined as SNR: $=10 \log _{10}\left(\sigma_{w}^{2} / \sigma_{v}^{2}\right)$.

As depicted by (1), the problem that we pose is to estimate the unknown phase parameters $\left(\theta, F_{e}\right.$ and $\left.\eta\right)$ of a random amplitude $(w(n))$ chirp signal embedded in unknown additive noise $(v(n))$, assuming knowledge of the received samples $\{x(n)\}_{n=0}^{N-1}$. The solution that we pursue consists of evaluating first certain generalized moments of the output that will remove the unwanted multiplicative effects introduced by the M-PSK modulated sequence $w(n)$. It turns out that the resulting problem reduces to the standard problem of estimating the phase parameters of a constant amplitude chirp signal embedded in additive noise, for which standard NLS estimators can be developed and their statistical properties analyzed in a rigorous manner.

\section{NONLINEAR CARRIER SYNCHRONIZER}

Consider the polar representation:

$$
x(n)=\rho(n) e^{j \varphi(n)},
$$

and define the process $y(n)$ via the nonlinear transformation:

$$
y(n):=F(\rho(n)) e^{j M \varphi(n)},
$$

where $F(\cdot)$ is in general a nonlinear function.

Conditioned on $w(n), x(n)$ is normally distributed with the probability density function (pdf) $f(x(n) \mid w(n)=2 \pi m / M, 0 \leq$ $m \leq M-1) \sim \mathcal{N}\left(w(n) \exp (j \phi(n)), \sigma_{v}^{2}\right)$. Throughout the paper, the notation $f(\cdot)$ will stand for the pdf of certain RVs. Due to (2), it follows that:

$$
\begin{aligned}
f(\rho(n), \varphi(n) \mid w(n)= & 2 \pi m / M)=\frac{\rho(n)}{\pi \sigma_{v}^{2}} e^{-\left(\rho^{2}(n)+1\right) / \sigma_{v}^{2}} \\
& \cdot e^{2 \rho(n) \cos [\varphi(n)-2 \pi m / M-\phi(n)] / \sigma_{v}^{2}}
\end{aligned}
$$


Based on (4), the joint and marginal pdf of $\rho(n)$ and $\varphi(n)$ take the expressions:

$$
\begin{gathered}
f(\rho(n), \varphi(n))=\frac{1}{M} \sum_{m=0}^{M-1} f\left(\rho(n), \varphi(n) \mid w(n)=\frac{2 \pi m}{M}\right) \\
=\frac{1}{M} \sum_{m=0}^{M-1} \frac{\rho(n)}{\pi \sigma_{v}^{2}} e^{-\frac{\rho^{2}(n)+1}{\sigma_{v}^{2}}} e^{\frac{2 \rho(n)}{\sigma_{v}^{2}} \cos \left[\varphi(n)-\frac{2 \pi m}{M}-\phi(n)\right]} \\
f(\rho(n))=\int_{-\pi}^{\pi} f(\rho(n), \varphi(n)) \mathrm{d} \varphi(n) \\
=\frac{2 \rho(n)}{\sigma_{v}^{2}} e^{-\left(\rho^{2}(n)+1\right) / \sigma_{v}^{2}} I_{0}\left(\frac{2 \rho(n)}{\sigma_{v}^{2}}\right)
\end{gathered}
$$

where $I_{0}(\cdot)$ stands for the zero-order modified Bessel function of the first kind [1, eq. (9.6.16)]. Using (5), some calculations show that:

$$
\begin{aligned}
& \mathrm{E}\{y(n)\}=\mathrm{E}\left\{F(\rho(n)) e^{j M \varphi(n)}\right\}=\mathcal{C} e^{j M \phi(n)}, \\
& \mathcal{C}:=|\mathrm{E}\{y(n)\}|=\mathrm{E}\left\{F(\rho(n)) \frac{I_{M}\left(\frac{2 \rho(n)}{\sigma_{v}^{2}}\right)}{I_{0}\left(\frac{2 \rho(n)}{\sigma_{v}^{2}}\right)}\right\},
\end{aligned}
$$

where $I_{M}(\cdot)$ denotes the M-order modified Bessel function of the first kind [1, eq. (9.6.19)], the expectation in (8) is with respect to (w.r.t.) the marginal distribution of $\rho(n)(6)$ and the resulting amplitude $\mathcal{C}$ is a real constant. Since $w(n)$ and $v(n)$ are i.i.d. and mutually independent, it follows that $u(n):=y(n)-\mathrm{E}\{y(n)\}$ is i.i.d., too. Consequently,

$$
y(n)=\mathcal{C} e^{j M \phi(n)}+u(n), n=0,1, \ldots, N-1,
$$

and $y(n)$ can be viewed as a constant amplitude chirp signal embedded in white noise. Note that in general, $u(n)$ is not circular.

Let $\boldsymbol{\omega}:=\left[\begin{array}{llll}\mathcal{C} & \omega_{0} & \omega_{1} & \omega_{2}\end{array}\right]^{T}=\left[\begin{array}{lllll}\mathcal{C} & M \theta & 2 \pi M F_{e} T & M \eta T^{2}\end{array}\right]^{T}$, and introduce the following NLS estimator (c.f. [3], [7]):

$$
\begin{aligned}
\hat{\boldsymbol{\omega}} & =\arg \min _{\overline{\boldsymbol{\omega}}} J(\overline{\boldsymbol{\omega}}), \\
J(\overline{\boldsymbol{\omega}}) & =\frac{1}{N} \sum_{n=0}^{N-1}\left|y(n)-\overline{\mathcal{C}} e^{j \sum_{l=0}^{2} \bar{\omega}_{l} n^{l}}\right|^{2} .
\end{aligned}
$$

After some algebra manipulations, the NLS estimates of $\omega_{l}, l=$ $0,1,2$ are obtained as [3]:

$$
\begin{aligned}
\left(\hat{\omega}_{1}, \hat{\omega}_{2}\right) & =\arg \max _{\bar{\omega}_{1}, \bar{\omega}_{2}} \frac{1}{N}\left|\sum_{n=0}^{N-1} y(n) e^{-j \sum_{l=1}^{2} \bar{\omega}_{l} n^{l}}\right|^{2} \\
\hat{\omega}_{0} & =\operatorname{angle}\left\{\sum_{n=0}^{N-1} y(n) e^{-j \sum_{l=1}^{2} \hat{\omega}_{l} n^{l}}\right\} .
\end{aligned}
$$

It is well-known that estimator (10) is asymptotically unbiased and consistent, and also almost asymptotically efficient at high SNR [3] and [5].

Following a procedure similar to the one presented in [3], one can derive the asymptotic variances avar $\left(\hat{\omega}_{l}\right):=N^{2 l+1} E\left(\hat{\omega}_{l}-\right.$ $\left.\omega_{l}\right)^{2}$ of $\hat{\omega}_{l}, l=0,1,2$, which are given by:

$$
\operatorname{avar}\left(\hat{\omega}_{l}\right)=\frac{\mathcal{B}-\mathcal{D}}{\mathcal{C}^{2}} \cdot \frac{1}{2 N^{2 l+1}} \cdot \frac{1}{2 l+1}\left[\frac{(l+3) !}{(l !)^{2}(2-l) !}\right]^{2}
$$

$$
\begin{aligned}
& \mathcal{B}:=\mathrm{E}\left\{|y(n)|^{2}\right\}=\mathrm{E}\left\{F^{2}(\rho(n))\right\}, \\
& \mathcal{D}:=\left|\mathrm{E}\left\{y^{2}(n)\right\}\right|=\mathrm{E}\left\{F^{2}(\rho(n)) \frac{I_{2 M}\left(\frac{2 \rho(n)}{\sigma_{v}^{2}}\right)}{I_{0}\left(\frac{2 \rho(n)}{\sigma_{v}^{2}}\right)}\right\} .
\end{aligned}
$$

Next, we choose an optimal or "matched" nonlinearity $F(\cdot)$ which minimizes the asymptotic variance (11). Since in (11), only $\mathcal{B}, \mathcal{C}, \mathcal{D}$ depend on $F(\cdot)$, finding an optimal $F(\cdot)$ resorts to solving the optimization problem:

$$
F_{\min }(\rho(n))=\arg \min _{F} \frac{\mathcal{B}-\mathcal{D}}{\mathcal{C}^{2}} .
$$

Using (8), (12) and (13), $F_{\min }(\cdot)$ is obtained using Cauchy-Schwarz' inequality and can be expressed as:

$$
F_{\min }(\rho(n))=\lambda \frac{I_{M}\left(\frac{2 \rho(n)}{\sigma_{v}^{2}}\right)}{I_{0}\left(\frac{2 \rho(n)}{\sigma_{v}^{2}}\right)-I_{2 M}\left(\frac{2 \rho(n)}{\sigma_{v}^{2}}\right)},
$$

where $\lambda$ is an arbitrary nonzero constant. The asymptotic variances of $\hat{\omega}_{l}, l=0,1,2$ corresponding to the matched nonlinearity (14) can be expressed as:

$$
\begin{aligned}
& \operatorname{avar}_{\min }\left(\hat{\omega}_{l}\right)=\frac{1}{2 N^{2 l+1}} \cdot \frac{1}{2 l+1} \cdot\left[\frac{(l+3) !}{(l !)^{2}(2-l) !}\right]^{2} \\
& \cdot \frac{1}{\mathrm{E}\left\{\frac{I_{M}^{2}\left(\frac{2 \rho(n)}{\sigma_{v}^{2}}\right)}{I_{0}^{2}\left(\frac{2 \rho(n)}{\sigma_{v}^{2}}\right)-I_{0}\left(\frac{2 \rho(n)}{\sigma_{v}^{2}}\right) I_{2 M}\left(\frac{2 \rho(n)}{\sigma_{v}^{2}}\right)}\right\}} .
\end{aligned}
$$

\section{MONOMIAL NONLINEAR ESTIMATORS}

As can be observed from (14), $F_{\min }(\rho(n))$ is a function that depends on the SNR. This is not a restrictive requirement since blind SNR estimators that exhibit good performance can be used. However, if estimating the SNR is not desirable, we show next that there exist optimal monomial approximations $\rho^{k}(n), k=0, \ldots, M$ of the matched nonlinearity $F_{\min }(\rho(n))$ that have almost the same asymptotic variance as (15) and their performance does not necessitate knowledge of the SNR.

It turns out that at high SNRs ( $\mathrm{SNR} \rightarrow \infty \mathrm{dB}$ ), based on $[8$, eq. (15)], the optimal monomial is $G_{h}(\rho(n))=\rho(n)$. Similarly, at low SNRs (SNR $\ll 0 \mathrm{~dB})$, based on [8, eq. (16)], the optimal monomial is $G_{l}(\rho(n))=\rho^{M}(n)$.

Define the class of processes $y_{k}(n), k=0, \ldots, N$, via the monomial transformations:

$$
y_{k}(n)=\rho^{k}(n) e^{j M \varphi(n)}, k=0, \ldots, M .
$$

Now it is interesting to study the asymptotic performance of the following class of NLS estimators:

$$
\hat{\boldsymbol{\omega}}^{(k)}=\arg \min _{\overline{\boldsymbol{\omega}}^{(k)}} \frac{1}{N} \sum_{n=0}^{N-1}\left|y_{k}(n)-\overline{\mathcal{C}} e^{j \sum_{l=0}^{2} \bar{\omega}_{l}^{(k)} n^{l}}\right|^{2},
$$

which can be viewed as a special case of (10) and whose asymptotic variances are given by:

$$
\begin{aligned}
& \operatorname{avar}\left(\hat{\omega}_{l}^{(k)}\right)=\frac{\mathcal{B}_{k}-\mathcal{D}_{k}}{\mathcal{C}_{k}^{2}} \frac{1}{2 N^{2 l+1}} \frac{1}{2 l+1}\left[\frac{(l+3) !}{(l !)^{2}(2-l) !}\right]^{2},(18) \\
& \mathcal{B}_{k}:=\mathrm{E}\left\{\left|y_{k}(n)\right|^{2}\right\}=\mathrm{E}\left\{\rho^{2 k}(n)\right\}, \\
& \mathcal{C}_{k}:=\left|\mathrm{E}\left\{y_{k}(n)\right\}\right|=\left|\mathrm{E}\left\{\rho^{k}(n) e^{j M \varphi(n)}\right\}\right|, \\
& \mathcal{D}_{k}:=\left|\mathrm{E}\left\{y_{k}^{2}(n)\right\}\right|=\left|\mathrm{E}\left\{\rho^{2 k}(n) e^{j 2 M \varphi(n)}\right\}\right| .
\end{aligned}
$$


Exploiting (6) and [6, eq. (6.643.4)], the following relation was derived in $[9,(\mathrm{~A} 17)]$ :

$$
\mathcal{B}_{k}=\sum_{q=0}^{k}\left(\begin{array}{l}
k \\
q
\end{array}\right)^{2} \sigma_{v}^{2 q} \cdot q !
$$

Using (6), we can also obtain that:

$\mathrm{E}\left\{y_{k}(n)\right\}=\frac{1}{\alpha^{k}} e^{j M \phi(n)} e^{-\frac{\gamma}{2}} \int_{0}^{\infty} \zeta^{k+1} e^{-\frac{\zeta^{2}}{2}} I_{M}(\alpha \zeta) \mathrm{d} \zeta$,

where: $\alpha:=\sqrt{2} / \sigma_{v}, \gamma:=\alpha^{2}$ and $\zeta:=\alpha \rho(n)$. Based on [6, eq. $(6.643,2)]$ and [1, eq. (13.1.32)], $\mathcal{C}_{k}$ can be expressed in terms of the confluent hypergeometric function $\Phi(\cdot, \cdot, \cdot)$ :

$$
\mathcal{C}_{k}=\frac{\Gamma\left(\frac{k+M}{2}+1\right) e^{-\frac{\gamma}{2}}}{\Gamma(M+1) \sigma_{v}^{M-k}} \Phi\left(\frac{k+M}{2}+1, M+1, \frac{\gamma}{2}\right) .
$$

Similarly,

$$
\mathcal{D}_{k}=\frac{\Gamma(k+M+1) e^{-\frac{\gamma}{2}}}{\Gamma(2 M+1) \sigma_{v}^{2 M-2 k}} \Phi\left(k+M+1,2 M+1, \frac{\gamma}{2}\right)
$$

Following a similar approach to that presented in [9], one can obtain a slightly more compact expression for the confluent hypergeometric function in (21):

$$
\begin{aligned}
\mathcal{D}_{k}= & \frac{1}{\gamma^{k}}\left[\gamma^{k} \sum_{p=0}^{M+k} p !\left(\begin{array}{c}
M+k \\
p
\end{array}\right)\left(\begin{array}{c}
M-k+p-1 \\
p
\end{array}\right)\left(\frac{-2}{\gamma}\right)^{p}\right. \\
& +(-1)^{M+k+1} 2^{k} e^{-\frac{\gamma}{2}}\left(\frac{2}{\gamma}\right)^{k+1} \sum_{p=0}^{M-k-1}\left(\begin{array}{c}
M+k+p \\
p
\end{array}\right) \\
& \left.\cdot \frac{(M+k) !}{(M-k-p-1) !}\left(\frac{2}{\gamma}\right)^{p}\right], \text { if } k=0,1, \ldots, M-1, \\
\mathcal{D}_{k}= & 1, \text { if } k=M .
\end{aligned}
$$

Plugging (19), (20) and (21) back into (18), a closed-form expression for the asymptotic variances $\operatorname{avar}\left(\hat{\omega}_{l}^{(k)}\right)$ is obtained for $k=0, \ldots, M$ and $l=0,1,2$. Note that at high SNR $(\rightarrow \infty \mathrm{dB})$, using [1, eq. (13.1.4)], some calculations show that:

$$
\lim _{\mathrm{SNR} \rightarrow \infty} \mathcal{C}_{k}=1
$$

for any $k=0,1, \ldots, M$. Hence, based on (18), (19), (22) and (23) we obtain:

$$
\lim _{\mathrm{SNR} \rightarrow \infty} \operatorname{avar}\left(\hat{\omega}_{l}^{(k)}\right) \propto M^{2} \frac{1}{\mathrm{SNR}},
$$

which does not depend on the estimator order $k$, i.e., it turns out that at high SNRs, the performance of estimators (17) for different orders $k$ is asymptotically the same.

\section{SIMULATION RESULTS}

In this section, we study thoroughly the performance of estimators (10) and (17) using computer simulations. The experimental mean-square error (MSE) results of (17) will be compared with the theoretical asymptotic bounds. The experimental results are obtained by performing a number of 200 Monte Carlo trials, the additive noise is generated as zero-mean Gaussian white noise with variance $\sigma_{v}^{2}$ and all the simulations are performed assuming the carrier phase $\theta=0.1$, frequency offset $F_{e} T=0.011$ and Doppler rate $\eta T^{2}=0.03$. There exist several methods to implement estimators (17). In this paper, we use the so-called high-order ambiguity function (HAF) approach, which has become a "standard" tool for analyzing constant amplitude chirp signals since it provides a computationally efficient yet statistically accurate estimator [3]. Due to space limit, we will not illustrate its details herein.

In this section, we also compare the asymptotic performance of proposed estimators w.r.t. the CRB for an unmodulated carrier, i.e., $M=1$, which is given as (c.f. [5]):

$$
\operatorname{CRB}\left(\hat{\omega}_{l}\right)=\frac{\sigma_{v}^{2}}{2 N^{2 l+1}} \cdot \frac{1}{2 l+1}\left[\frac{(l+3) !}{(l !)^{2}(2-l) !}\right]^{2} .
$$

Experiment 1-Performance loss of estimators (16)-(17) w.r.t. the matched estimator (14)-(10): Figs. 1-2 plot the loss in performance of estimators (16)-(17) w.r.t. the optimal nonlinearity estimator (14)-(10) $\left(-10 \log _{10}\left[\operatorname{avar}\left(\hat{\omega}_{l}^{(k)}\right) / \operatorname{avar}_{\min }\left(\hat{\omega}_{l}\right)\right]\right)$ in the case of a BPSK modulation $(M=2)$ and QPSK modulation $(M=4)$, respectively. It turns out that in almost the entire SNR region of interest, the optimal nonlinearity $F_{\min }(\rho(n))$ can be approximated without much loss in performance by $\rho(n)$ (BPSK) and $\rho^{2}(n)$ (QPSK), respectively.

Experiment 2-Asymptotic variances of estimators (14)-(10) and (16)-(17) w.r.t. CRB: Fig. 3 illustrates the theoretical asymptotic variances of estimators (10) and (17) versus SNR. The theoretical asymptotic variances are compared with the CRB. Fig. 3 depicts the performance loss of the asymptotic variances (15) and (18) w.r.t. CRB (i.e., $-10 \log _{10}\left[\operatorname{avar}\left(\hat{\omega}_{l}^{(k)}\right) / \operatorname{CRB}\left(\hat{\omega}_{l}\right)\right]$ ), assuming a QPSK modulation. It can be seen that the proposed estimators exhibit good accuracy. In high SNR range they coincide with the CRB. In low SNR range (near OdB), we can improve the performance of estimators (17) by adopting low order estimators $(k=1$ and 2). Although the optimal nonlinearity estimator has the best performance in the entire SNR range, the improvement is minor. From Figs. 1-3, we can also observe that at high SNRs, the monomial estimators (16)-(17) for different orders $k$ exhibit the same asymptotic variance.

Experiment 3-Comparison of MSE of estimators (17) with the theoretical bounds: In Figs. $4-6$, the theoretical bounds (18) are compared with experimental MSEs of the estimators (17). The results are plotted versus SNR, assuming $N=50$, BPSK modulation. These figures show that for medium and high SNR, the experimental results are well predicted by the asymptotic bounds derived in Section 4, and the proposed estimators provide very good estimates of carrier phase, frequency offset and Doppler rate, even when a reduced number of samples are used $(N=50)$. This shows the potential of these estimators for fast synchronization of burst transmissions.

\section{CONCLUSIONS}

In this paper, we have introduced and analyzed a family of blind feedforward joint carrier phase, frequency offset and Doppler rate estimators for burst-mode M-PSK modulations. A matched nonlinear estimator together with a class of monomial nonlinear estimators were introduced and their performance established.

Acknowledgement: This work was supported by the NSF Career Award No. CCR-0092901. 


\section{REFERENCES}

[1] M. Abramowitz and I. A. Stegun, Eds., Handbook of Mathematical Functions, Washington, DC: NBS 1964.

[2] S. Bellini, "Frequency estimators for M-PSK operating at one sample per symbol," GLOBECOM, 1994.

[3] O. Besson, M. Ghogho and A. Swami, "Parameter estimation for random amplitude chirp signals," IEEE Trans. Signal Processing, vol. 47, no. 12, pp. 3208-3219, Dec. 1999.

[4] F. Classen, H. Meyr, and P. Sehier, "Maximum likelihood open loop carrier synchronizer for digital radio," ICC, 1993.

[5] M. Ghogho, A. K. Nandi and A. Swami, "Cramèr-Rao bounds and maximum likelihood estimation for random amplitude phase-modulated signals," IEEE Trans. Signal Processing, vol. 47, no. 11, pp. 2905-2916, Nov. 1999.

[6] I. S. Gradshteyn and I. M. Ryzhik, Table of Integrals, Series, and Products, New York: Academic, 1965.

[7] F. Mazzenga and G. E. Corazza, "Blind least-squares estimation of carrier phase, Doppler shift, and Doppler rate for M-PSK burst transmission," IEEE Communications Letters, vol. 2, no. 3, pp. 73-75, 1998.

[8] B. E. Paden, "A matched nonlinearity for phase estimation of a PSK-modulated carrier," IEEE Trans. Information Theory, vol. 32, no. 3, pp. 419-422, May 1986.

[9] A. J. Viterbi and A. M. Viterbi, "Nonlinear estimation of PSK-modulated carrier phase with application to burst digital transmissions," IEEE Trans. Information Theory, vol. 29, no. 4, pp. 543-551, July 1983.

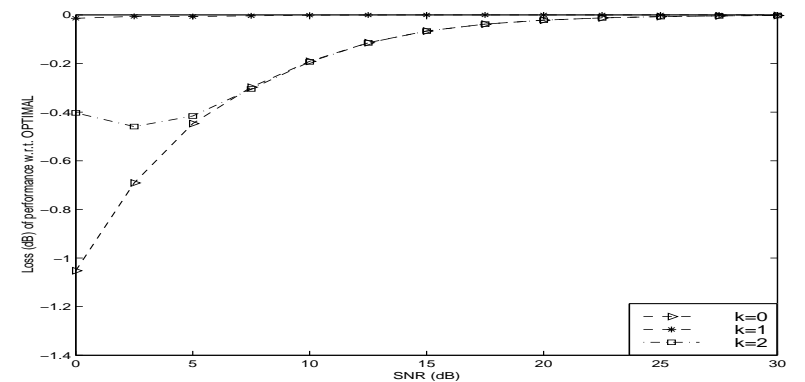

Fig. 1. Theoretical degradation of $\hat{\omega}_{l}^{(k)}$ w.r.t. optimal nonlinearity versus SNR for BPSK constellation.

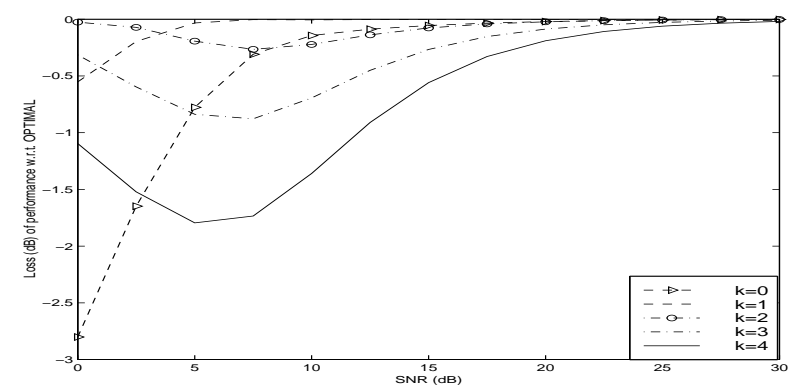

Fig. 2. Theoretical degradation of $\hat{\omega}_{l}^{(k)}$ w.r.t. optimal nonlinearity versus SNR for QPSK constellation.

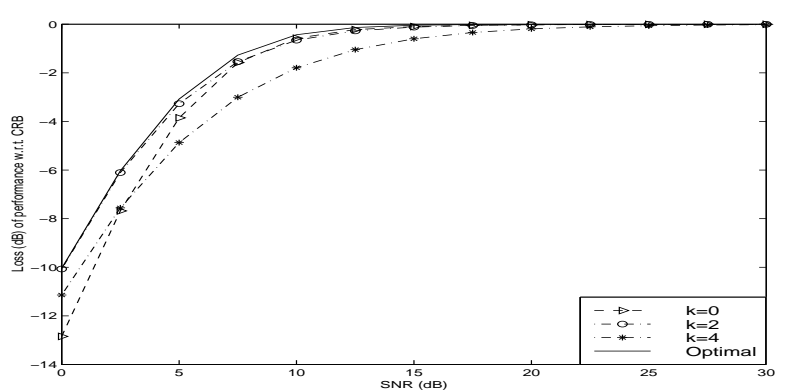

Fig. 3. Performance loss w.r.t. CRB versus SNR for QPSK modulation.

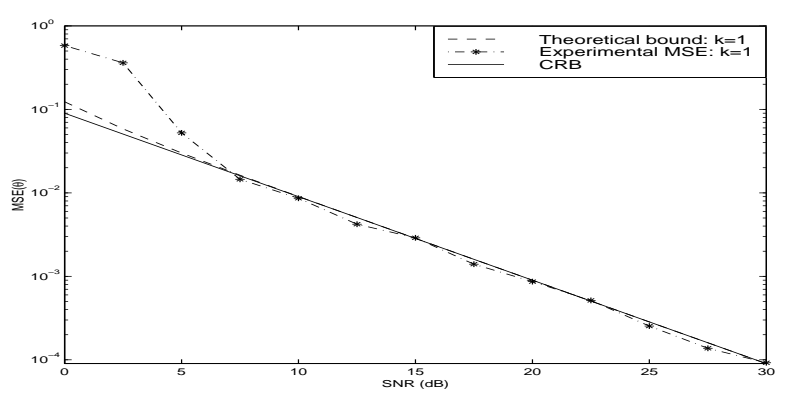

Fig. 4. MSEs of $\widehat{\theta}$ versus SNR for BPSK modulation.

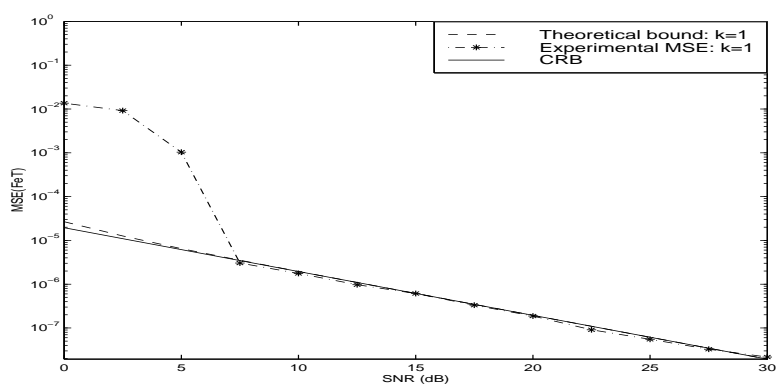

Fig. 5. MSEs of $\widehat{F_{e} T}$ versus SNR for BPSK modulation.

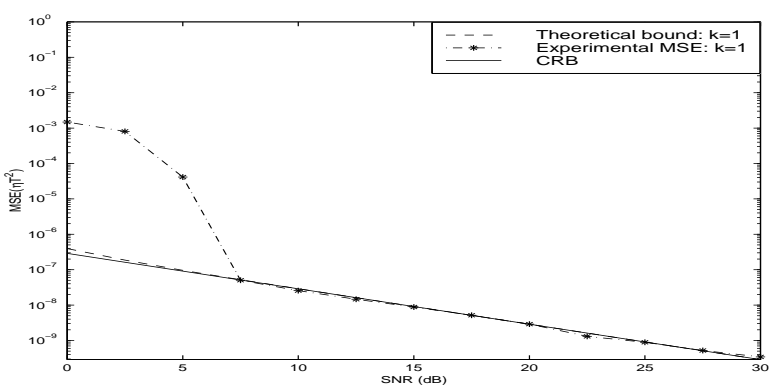

Fig. 6. MSEs of $\widehat{\eta T^{2}}$ versus SNR for BPSK modulation. 